\title{
Mechanical stretch regulates hypertrophic phenotype of the myometrium during pregnancy
}

\author{
Oksana Shynlova ${ }^{1}$, Ruth Kwong ${ }^{1,2}$ and Stephen J Lye $\mathrm{e}^{1,2,3}$ \\ ${ }^{1}$ Samuel Lunenfeld Research Institute, Mount Sinai Hospital, 25 Orde Street, Room 6-1019, Toronto, Ontario, Canada \\ M5T $3 \mathrm{H} 7$ and Departments of ${ }^{2}$ Physiology and ${ }^{3}$ Obstetrics and Gynecology, University of Toronto, Toronto, Ontario, \\ Canada M5S 1A1
}

Correspondence should be addressed to O Shynlova; Email: shynlova@lunenfeld.ca

\begin{abstract}
The adaptive growth of the uterus is a critical event that involves changes in cellular phenotypes throughout pregnancy. In early pregnancy, uterine growth is due to hyperplasia of uterine smooth muscle cells (SMCs) within the myometrium; however, the major component of myometrial growth occurs after mid-gestation. This study sought to test the hypothesis that increase in myometrial growth seen during late pregnancy is due to SMC hypertrophy caused by mechanical stretch of uterine tissue by a growing fetus(es) by providing direct measurements of individual SMC size. We employed a stereological approach to calculate the average cell volumes of uterine myocytes through diameter measurements using the Stereoinvestigator statistical software. Uterine tissues were collected from nonpregnant Wistar rats, as well as from gravid and nongravid horns of unilaterally pregnant animals on gestational days (d) 8 (early gestation), 14 (mid-gestation), 19 (late gestation), 22 (term), and 4 days post partum. Anti-caveolin-1 immunostaining was used to clearly delineate SMC boundaries. The stereological analysis revealed that the dramatic increase in myometrial growth seen during late gestation (d19-22) is due to a threefold increase in the size of uterine myocytes. A significant increase in SMC volumes was detected in the gravid uterine horn as compared with the corresponding empty horn of unilateral term pregnant animals (day 22, mean cell volume $\left.1114 \mathrm{vs} 361 \mu \mathrm{m}^{3}, P<0.05\right)$, indicating the effect of uterine occupancy. The restriction of the hypertrophy to cells within the gravid horn suggests that it may be a response to the biological mechanical stretch of uterine walls by the growing fetus(es) and placenta(s).

Reproduction (2010) 139 247-253
\end{abstract}

\section{Introduction}

Uterine smooth muscle undergoes dramatic physiological adaptations during the course of pregnancy. We described earlier two distinct phases of myometrial growth from early to late pregnancy: 1) myocyte hyperplasia associated with an increase in anti-apoptotic proteins in the first half of gestation ('proliferative' phase) and 2) myometrial hypertrophy associated with the increase in smooth muscle cell (SMC) size ('synthetic' phase) in the second half of gestation (Shynlova et al. 2006). Cellular hypertrophy is characterized by an increase in cellular protein and is associated with increased thickness of uterine muscle layers, as well as with significant changes in mass and composition of extracellular matrix, the tension-bearing components of the uterus (Harkness \& Harkness 1954, Gabella 1990, Shynlova et al. 2004).

Despite the remarkable nature of uterine growth during pregnancy, there is little information as to the mechanisms that initiate and regulate these two growth phases. We have recently discovered that activation of the IGF1/PI3K/MTOR signaling pathway in uterine myocytes by estrogen is responsible for the induction of myometrial hyperplasia during the proliferative phase of gestation (Jaffer et al. 2009). However, few potential mechanisms regulating myometrial hypertrophy have been proposed in the literature. It was reported that ovarian hormones, estrogen and progesterone, were able to induce myometrial hypertrophy (Martin et al. 1973, Koseki \& Fujimoto 1974). Several groups in the past have also suggested that in addition to endocrine factors, the enlarging fetus might induce uterine growth by stretching the uterine wall (Csapo et al. 1965, Douglas et al. 1988). The experimental uterine distension by saline fluid-filled balloon was applied into nonpregnant (NP) uterine horns to mimic the mechanical stretch induced during pregnancy (Douglas et al. 1988). This study demonstrated an increased uterine growth due to a combination of both hypertrophy and hyperplasia (Douglas et al. 1988). However, the NP model does not account for the effect of hormonal regulation unlike the unilateral pregnant model that provides an internal control separating hormonal effects from mechanical influences. Using unilaterally pregnant rats, Goldspink \& Douglas (1988) reported that at mid-gestation 
protein:DNA ratio, a biochemical marker of hypertrophy, was dramatically increased in the gravid horn (subjected to stretch by growing fetus), while remaining unchanged in the nongravid horn. These studies confirm that mechanical stretch plays an important and, perhaps, dominant role in promoting the growth of the uterus during pregnancy. We studied protein:DNA ratios in pregnant rat myometrium throughout gestation and showed a marked increase in cell size during the second half of gestation, when fetal growth is maximal (Shynlova et al. 2006). This result indirectly indicated that mechanical stretch exerted by the growing fetus might be one of the main signals that cause the uterine myocytes to shift from the proliferative to the synthetic phase of myometrial growth. However, the ratio is an indirect method and there are currently no real numerical measurements of uterine cell volumes throughout gestation to more accurately portray the degree of growth in the myometrial SMCs. In the context of the uterus, this measurement may underestimate the true degree of myometrial hypertrophy, as polyploidal nuclei appear in the myometrium during pregnancy (van der Heijden \& James 1975). This is especially true for further study of physiological mechanisms originating and regulating myometrial hypertrophy, which require a more reliable and direct measure of cell volumes. The elucidation of the numerical measurement of cell growth will also provide a better context to test the validity of previously proposed mechanisms.

Thus, the goal of the present study was 1) to apply stereological methodology to elucidate a direct measurement of uterine SMCs volume throughout gestation and 2) to assess the contribution of mechanical signal to the maintenance of the synthetic myometrial phenotype. Firstly, using a computerized stereological approach, we elucidated statistical proof for the existence of myometrial hypertrophy during normal bilateral pregnancy. The methodology applied in this research is three-dimensional statistical analysis software, the Stereoinvestigator (MicroBrightField, Inc., Williston, VT, USA), which can calculate cell volumes by using the point sample intercept (PSI) microscope probe. In order to use the Stereoinvestigator, the membranes of individual myocytes must be clearly visible to mark the cell boundaries for the diameter measurements and consequent volume calculations to be done. Therefore, we applied anticaveolin (CAV) 1 and 3 antibodies to visualize plasma membrane of individual SMCs. Secondly, to assess our hypothesis that restriction of myocyte hypertrophy to the gravid horn is due to myometrial stretch of the uterine wall by growing fetus(es), we used the unilateral pregnant rat model. The isotropic uniform random (IUR) sampling was used for tissue collection (see Material and Methods). The resulting cell diameter measurements collected allow average cell volumes to be calculated for NP, early, mid, late, term pregnant, and post partum (PP) uterine samples showing statistically reliable data and proving the occurrence of myometrial hypertrophy at late gestation.

\section{Results}

\section{Antibody selection for immunohistochemical staining}

In order to measure the size of myometrial cells using the Stereoinvestigator approach, the individual SMC boundaries must be clearly visible. CAV1, 2, and 3 are transmembrane proteins universally expressed in the small omega-shaped invaginations of plasma membrane called caveolae. CAV1 appears to be a principal protein component of caveolal membrane in vivo. It was reported recently that CAV1 was expressed at a constant level in mouse myometrium throughout gestation $\mathrm{Wu}$ et al. 2008). CAV3 represents the muscle-specific member of the CAV supergene family, as its expression is limited to striated and SMCs (Couet et al. 2001). Therefore, antiCAV1 and anti-CAV3 antiserum were used for immunohistochemistry analysis to find the antibody that provides the best quality immunostaining and is less susceptible to changes during the gestational period. When rat uterine tissues were immunostained with anti-CAV1 antiserum, strong positive signals were detected in plasma membranes of all SMCs in both longitudinal and circular myometrial layers (Fig. 1). Analysis of CAV3 immunostaining revealed that this protein was localized to the cell membrane of all samples studied, although the staining was very weak (data not shown). Based on these results, anti-CAV1 antibodies were selected for stereological analysis of myometrial samples from bilaterally (Fig. 1) and unilaterally (Fig. 2) pregnant animals.

\section{Cellular hypertrophy during normal gestation}

Myometrial tissue sections from three different sample sets ( $n=3$ per gestational day) were stained for CAV1 protein to visualize the plasma membrane of SMCs. The stereological analysis confirmed that the dramatic increase in uterine size seen during late gestation is due to an increase in the size of individual uterine myocytes. Representative images showed little change in cell size from NP up to day 14 of pregnancy (Figs 1 and $3 \mathrm{~A}$ ). The mean cell volumes were similar between the NP (264 $\pm 112 \mu^{3}$ ) and early pregnant uterus (day 8, 245 $\pm 90 \mu^{3}$ ). However, myometrial hypertrophy increased noticeably with gestation. From day 14 to day 19 , a significant increase in SMCs size occurred ( $448 \pm 225$ vs $\left.771 \pm 402 \mu \mathrm{m}^{3}, P<0.05\right)$, uterine myocytes remained hypertrophic until term (day 22, 716 $\pm 364 \mu \mathrm{m}^{3}$ ) and then dramatically decreased by 4 days PP (4PP; 296 $\pm 101 \mu \mathrm{m}^{3}$; Figs 1 and $3 \mathrm{~A}$ ). There was no difference between the size of myometrial cells on day 19 and day 22, confirming our earlier observations that uterine growth stabilizes at term. 

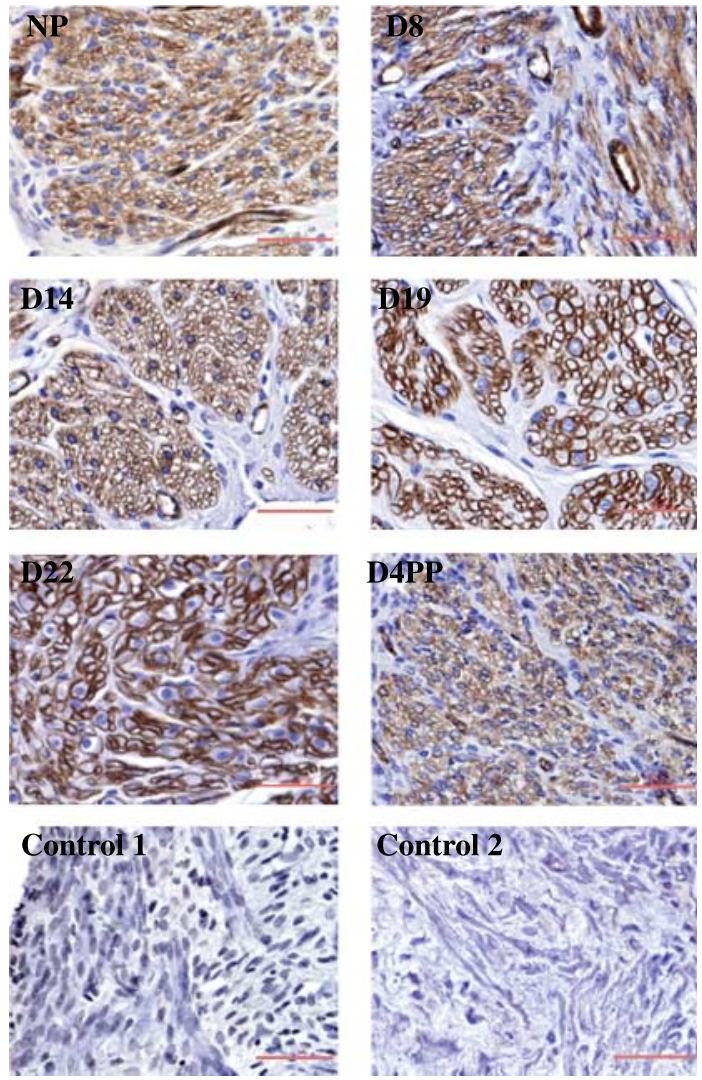

Figure 1 Caveolin-1 immunolocalization in nonpregnant, bilaterally pregnant rat myometrium during gestation and post partum. Immunohistochemical examination was performed on sections of uterus from nonpregnant (NP), day 8, day 14, day 19, and day 22 of pregnancy and 4 days post partum (4PP) animals. Negative controls were performed by either omitting primary $\mathrm{Ab}$ or nonspecific goat IgGs. For each day of gestation, tissue was collected from three different animals. Original magnification is $400 \times$; scale bars, $50 \mu \mathrm{m}$.

\section{Effect of biological stretch on myometrial hypertrophy}

Hypertrophic changes in the myometrium were determined by measuring the volume of individual SMCs at four different gestational time points in both gravid and nongravid uterine horns. Mean cell volume increased dramatically with gestation in the gravid horn only, while no change in cellular volume was observed in the nongravid horn (Figs 2 and 3B). Myometrial hypertrophy was significantly higher in gravid uterine horn compared with the empty horn of unilaterally pregnant animals at late gestation (day 19, 1023 vs $351 \mu \mathrm{m}^{3}, P<0.05$ ) and at term (day 22, mean cell volume 1114 vs $\left.361 \mu \mathrm{m}^{3}, P<0.05\right)$, indicating the effect of uterine occupancy. The restriction of hypertrophy to cells within the gravid horn suggests that it is a response to the passive biological mechanical stretch of uterine walls by the growing fetus(es) as both horns develop in the same hormonal environment.

\section{Discussion}

In the present study, we tested the hypothesis that the increase in myometrial growth seen during late pregnancy is due to SMC hypertrophy caused by mechanical stretch of uterine tissue by growing fetus(es). Using stereological analysis of uterine tissue sections, we 1) provide direct measurements of individual SMC size throughout gestation and 2) demonstrate that myometrial hypertrophy occurred only in the gravid horn of unilaterally pregnant rats.

Over the last 30 years, the protein:DNA ratio was a commonly used marker to identify cellular hypertrophy in different tissues (Johansson 1984). This widely accepted method is an indirect biochemical representation of the increase in cellular size by changes in protein content relative to the change in DNA content. Csapo et al. (1965) used this methodology to estimate
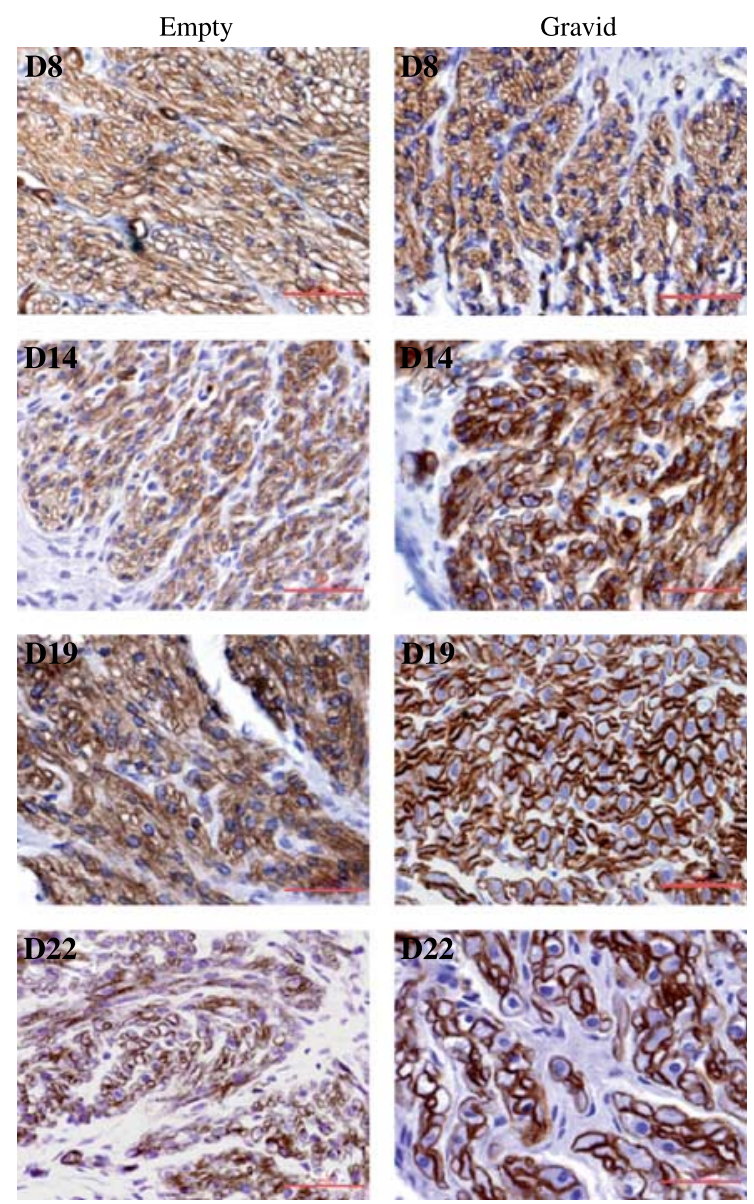

Figure 2 The diameter of individual myometrial cells increases only in the gravid horn of unilaterally pregnant rats. Uterine tissues were collected from empty and gravid horns of unilaterally pregnant rats on different gestational days, including day 8 , day 14 , day 19 , and day 22 . Tissues were labelled with anti-caveolin- 1 antibody and light microscopy images of cross sections were collected. Caveolin-1 was localized strongly within the plasma membrane of individual SMCs in the gravid uterine horn and showed a weaker staining in the empty horn. Magnification is $400 \times$; scale bar, $50 \mu \mathrm{m}$. 

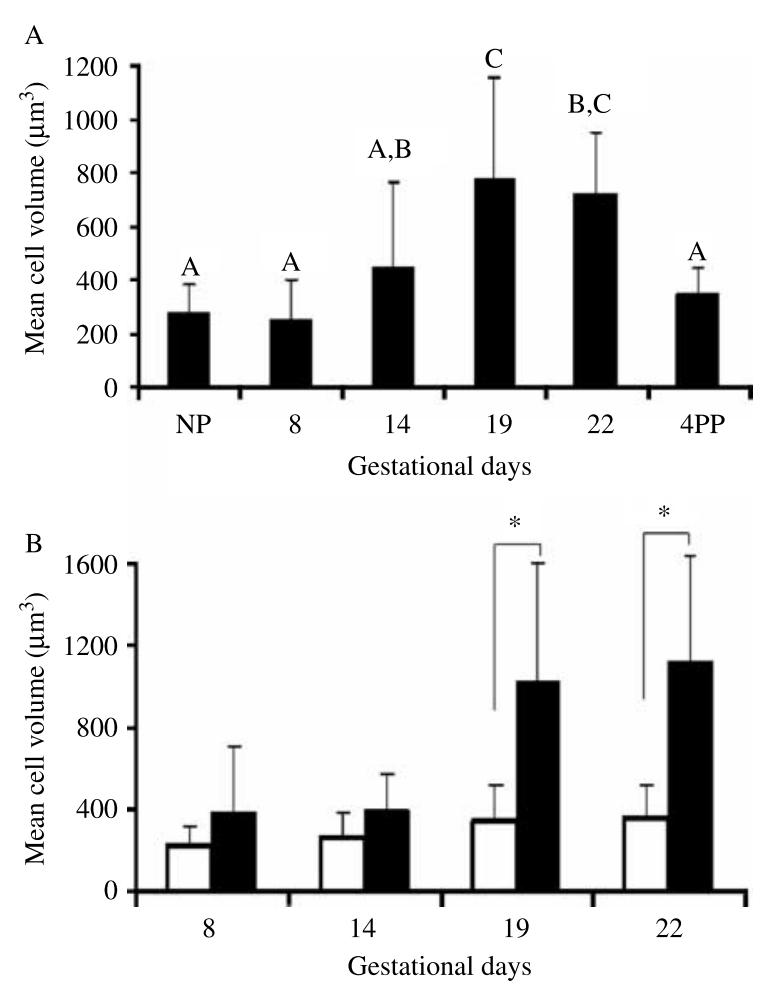

Figure 3 Average volume of myometrial SMCs in bilaterally pregnant (A) and unilaterally pregnant (B) rats. (A) SMC volume in the pregnant rat myometrium increases at late gestation. Data labelled with different letters are significantly different from each other $(P<0.05)$. (B) Mechanical stretch stimulates the increase in average cell volume only in gravid (black bars) but not in the empty (white bars) horn myometria of unilaterally pregnant rats. A significant difference from the empty horn of the same gestational day is indicated by $*(P<0.05)$. The bar graphs show the mean cell volume \pm s.D. ( $n=361-724$ at each time point).

the extent of myometrial growth in uterine horn of ovariectomized rabbits inflated with a saline-filled balloon (Csapo et al. 1965). Later an increase in protein:DNA ratio was demonstrated in NP uterine horn stretched by a saline-filled balloon indicating the induction of hypertrophy (Douglas et al. 1988). The injected fluid, however, does not fully imitate the shape of the growing fetus and the dynamic of the gestational process. Using unilaterally pregnant rats, Goldspink \& Douglas (1988) showed that stretched gravid horns promoted uterine hypertrophy on day 14 and 16 of gestation, while nongravid horns of these rats experienced no growth. Interestingly, the region of the uterus immediately surrounding the enlarging fetus and, consequently, experiencing the most significant degree of stretch demonstrated greater cellular hypertrophy (protein:DNA ratio) compared with regions in between fetuses that were subjected to less mechanical stretch (Goldspink \& Douglas 1988). However, the appearance of polyploidal nuclei in the myometrium during pregnancy (van der Heijden \& James 1975) makes the measurement of DNA content a somewhat unreliable indicator of cellular hypertrophy. The presence of polyploidal nuclei would result in a smaller protein:DNA ratio and may underestimate the true degree of myometrial hypertrophy during gestation.

Until now, however, no numerical measurements of uterine cell volume were recorded to accurately describe gestational changes of the myometrial SMCs. The alternative method for the study of myometrial hypertrophy is stereological analysis, which allows direct measurement of individual cell diameters using histological sections of uterus collected during different phases of gestation. Based on the individual cell diameter, myometrial cell volumes are calculated automatically by the computer using three-dimensional statistical analysis software, Stereoinvestigator. The randomized procedure of tissue sampling allows many different intercept measurements of the cells to be collected, yielding an unbiased average cell volume for each gestational day studied. Myometrial hypertrophy was low at the beginning of pregnancy, increasing dramatically with gestational age and was partially reversed after 4PP (Figs 1 and 3). In accordance with our previous results, we recorded a statistically significant threefold increase in myometrial cell volume at late gestation as compared with NP samples. Although we and other investigators have shown earlier hypertrophic changes of uterine myocytes, this study is the first to directly measure the volume of myometrial SMCs throughout pregnancy. Importantly, this hypertrophic growth was only present in the gravid horn and not the empty horn of unilaterally pregnant rats, suggesting that passive biological mechanical stretch due to fetal growth may be the signal for hypertrophy. Importantly, mechanical forces were shown to be essential to stimulate hypertrophy of vascular, interstitial, and urinary bladder SMCs and cardiac myocytes. Chronic outflow obstruction of rat and mini-pig urinary bladder caused similar (2.5 fold) increase in SMC size (Lindner et al. 1988, Nielsen et al. 1995). Analogous to a reverse of myometrial hypertrophy during the PP involution period, removal of the obstructing ligature was followed by a partial reversal of bladder cell hypertrophy (Lindner et al. 1988). In addition, experimental stenosis of rat small intestine caused an up to tenfold increase in muscle volume due to an increase in both size and number of intestinal SMCs (Johansson 1984). The experimental portal hypertension caused a hypertrophy of vascular SMCs (Johansson 1984), accompanied by development of polyploidy in a large fraction of the cells (Owens 1989).

We used CAV1 protein as a gestation-independent (Wu et al. 2008) specific marker of plasma membrane to clearly identify boundaries of individual myometrial cells. Recent studies have implicated lipid-rich membrane caveolae in mechanosensing (Rothberg et al. 1992, Park et al. 2000, Sunaga et al. 2004, Bailey \& Liu 2008). Caveolae, 50- to $100-n m$ flask-shaped invaginations of the cell membrane, are biochemically distinct from other parts of the cell membrane as they contain 
large amounts of cholesterol and sphingolipids as well as CAV proteins. These maintain the shape of caveolae but also act as scaffolds for several intracellular signaling proteins (for reviews, see (Hayashi et al. 2001, Bailey \& Liu 2008)). It is within this organelle that cells perform transmembrane trafficking and signal transduction. Caveolae are particularly abundant in muscle cells, adipocytes, fibroblasts, endothelial cells and epithelial cells. All three isoforms of CAV (CAV1, 2 and 3) are expressed in SMCs, with CAV1 being the quantitatively dominating homolog (Campbell et al. 2001, Couet et al. 2001). It has been reported recently that focal adhesion kinase (FAK), the major regulator of cell-ECM contacts, can up-regulate CAV1 expression in human embryonic cancer cell lines. In addition, examination of $\mathrm{FAK}^{+/+}$ and $\mathrm{FAK}^{-1-}$ mouse embryonic fibroblasts revealed that in cells devoid of FAK, CAV1 expression is strikingly diminished (Bailey \& Liu 2008). Macphee \& Lye (2000) discovered earlier that FAK expression and activity was up-regulated in rat myometrium at late gestation. It is well known that activation of FAK causes the formation of complex specialized structures termed focal adhesions, associated with the plasma membrane of SMCs. Integrin receptors are essential components of focal adhesions. CAV1 was found in complexes with $\beta 1$ integrin. CAV binds cholesterol and several signaling molecules potentially linked to integrin function, e.g. Src family kinases. It was reported that ligand-induced signaling necessary for normal $\beta 1$ integrin function requires CAV (Wei et al. 1999). We reported recently that both fibronectin and its integrin receptor $\alpha 5 \beta 1$ have been up-regulated at late gestation, specifically in the gravid uterine horn of unilaterally pregnant rat. We speculate now that CAV1 protein in myometrial SMCs may positively affect integrin-mediated FAK signaling.

We have shown earlier that in addition to hormones, myometrial stretch contributes to the expression of a cassette of genes (i.e. gap junctions, agonist receptors) required to activate the myometrium in order to initiate labour contraction. Although this biological stretch is exerted on the uterine wall by the growing fetus and placenta throughout pregnancy, these genes are not turned on during gestation, but instead the process occurs just before term. We speculate now that during pregnancy and prior to labour, uterine growth created by hypertrophy provides a mechanism to prevent an increase in tension on the myometrium and thus maintain uterine quiescence. The current study sheds light upon the potential mechanism regulating hypertrophic uterine growth.

\section{Material and Methods}

\section{Animals}

Wistar rats (Charles River Co., St Constance, Quebec, Canada) were housed individually under standard environmental conditions (12 h light:12 h darkness cycle) and fed Purina
Rat Chow (Ralston Purina, St Louis, MO, USA) and water ad libitum. Female virgin rats were mated with male rats and day 1 of gestation was designated as the day a vaginal plug was observed. The average time of delivery was during the morning of day 23. The Samuel Lunenfeld Research Institute Animal Care Committee approved all animal experiments.

\section{Experimental design}

\section{A. Normal pregnancy and term labor}

Animals were killed by carbon dioxide inhalation and uterine samples were collected on gestational days 0 (NP), 8, 14, 19, 22, and 4PP.

\section{B. Unilaterally pregnant rats}

Under general anesthesia, virgin female rats underwent tubal ligation through a flank incision to ensure that they subsequently became pregnant in only one horn (Ou et al. 1998). Animals were allowed to recover from surgery for at least 7 days before mating. Pregnant uterine samples from empty and gravid horns were collected on days 8, 14, 19, and 22. For each day of gestation, tissue was collected from three different animals $(n=3)$.

\section{Tissue collection}

Stereological investigation required an IUR sampling method for sampling a structure in which all orientations in three dimensions have equal weight (Martin et al. 1973, Gundersen $\&$ Jensen 1985, Mouton 2002). The intact uterine horns were placed in ice-cold PBS buffer and cut into 3-10 mm segments using a scalpel blade. Uterine tissue samples were collected at regular intervals from a random starting point on each uterine horn (every other pup in each gravid horn or every other uterine segment from each empty horn) and fixed immediately in $4 \%$ paraformaldehyde (PFA) solution at $4{ }^{\circ} \mathrm{C}$ for $24 \mathrm{~h}$. The PFA-fixed uterine tissue segments were then gradually dehydrated in ethanol and provided further random orientation by randomly embedding all tissue segments from the same gestational day in individual paraffin blocks (Fig. 4).

\section{Immunohistochemistry}

Tissue samples were immunostained using anti-CAV1 and antiCAV3 antibodies to visualize plasma membrane of individual SMCs. Multiple sections of the uterine horn (5 $\mu \mathrm{m}$ thickness) were collected on Superfrost Plus slides (Fisher Scientific, Nepean, ON, Canada) depending on the randomized starting location and length of uterine horn. Paraffin sections were deparaffinized and rehydrated. Endogenous peroxidase activity was inhibited with hydrogen peroxide $(5 \%$ in methanol) for $10 \mathrm{~min}$, and sections were retrieved in $0.125 \%$ trypsin solution at RT for $35 \mathrm{~min}$, blocked with DAKO Protein Serum-Free Blocking solution (Dako Corporation, Carpinteria, CA, USA) for $1 \mathrm{~h}$ and incubated overnight with goat polyclonal anti-CAV1 (1:500 for NP, d8 and d4PP and 1:1000 for d14, d19, d22; Cell Signalling Inc., Beverly, MA, USA) or mouse 


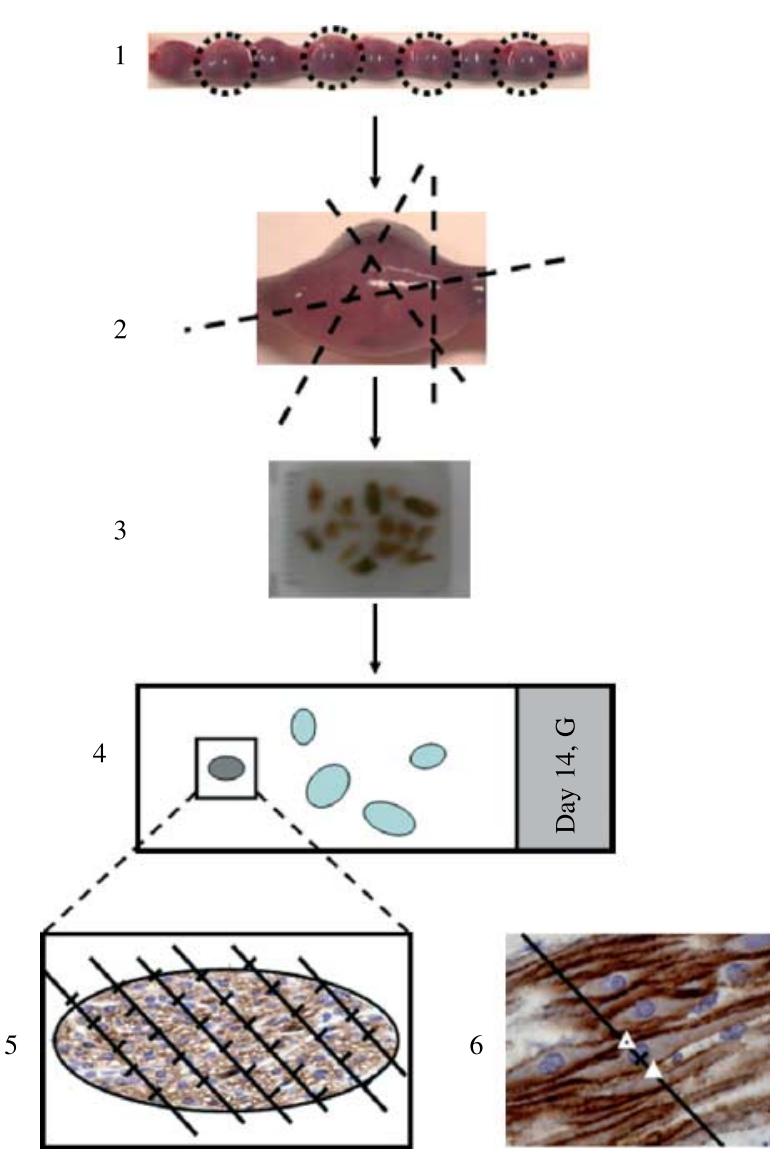

Figure 4 Isotropic uniform random (IUR) sampling procedure. (1) Uterine tissue samples were collected at regular intervals from a random starting point on each uterine horn (every other pup in the gravid horn or every other segment of the empty horn). (2) Collected tissues were cut in a random orientation. (3) Multiple uterine tissues samples from same gestational day were embedded in a random orientation in individual paraffin blocks. (4) Tissue samples were immunostained using anti-caveolin- 1 antibodies to visualize plasma membrane of individual SMCs. (5) Stereoinvestigator software generates sets of lines overlaying the entire tissue slide. (6) Boundaries of individual cells where an intercept (line) runs through were identified and marked (white triangles) to determine the diameters of these myocytes.

anti-CAV3 (1:200; Santa Cruz Biotechnology Inc., Santa Cruz, CA, USA) primary antibodies. For the negative controls, ChromPure nonspecific goat IgGs (Santa Cruz) were used at the same concentration as primary antibodies, or PBS was applied instead of the primary antibody. Secondary antibodies used were biotinylated anti-goat (1:200, Dako Corporation) and biotinylated anti-mouse (1:200; Dako). Visualization was achieved using LSAB Kits and HRP-conjugated streptavidin (DakoCytomations, Inc). Counterstaining with Harris Hematoxylin (Sigma Diagnostics) was carried out before slides were mounted with Cytoseal XYL (Richard-Allan Scientific, Kalamazoo, MI, USA). Myometrial cells from each set of tissues were observed on a Leica DMRXE microscope (Leica Microsystems, Richmond Hill, ON, Canada), and representative tissue sections were photographed with a Sony DXC-970 MD (Sony Ltd, Toronto, ON, Canada) 3CCD color video camera.

\section{Stereological analysis}

The average myometrial cell volume during gestation was estimated using a Leica DMRB microscope equipped with a DVC colour digital camera, which was connected to a Dell Precision 450 workstation and Stereoinvestigator software package (MicroBrightfield) from Wright Cell Imaging Facility in Toronto Western Hospital. The live image of each antiCAV1-stained slide that was placed under the microscope was presented onto the workstation where the area of interest was traced and PSI probe was applied. This tool in the Stereoinvestigator generates a series of uniform random test lines, under random angle, and overlays the entire histological sample such that only individual SMCs, where marked points of test lines landed, were selected for analysis (see Fig. 4). Boundaries of these individual cells where intercept (line) runs through were identified and recorded using a virtual marker tool. Intercept measurements that are utilized for cell volume calculations represent intervals (in $\mu \mathrm{m}$ ) between two marks. The entire area of interest was systematically scanned using the Meander Scan option. The randomized procedures allow many different intercept measurements of the cells to be collected, yielding an unbiased average cell volume for each gestational day studied (Gundersen \& Jensen 1985, Mayhew 1989). Cell volume was calculated by the software (Stereo Investigator 7, Document Version 4.00). All measurements were made at $200 \times$ magnification and $\sim 20-40$ fields of view per uterine sample were analyzed, producing a final mean volume of myocytes estimated from 250-650 cells/gestational day. The coefficient of error (CE; Gundersen \& Jensen 1987) was estimated for each case. For normal pregnancy, mean CE was 0.038 (range: 0.023-0.05); for unilateral pregnancy, mean CE was 0.03846 (range: $0.027-0.05)$.

\section{Statistical analysis}

Individual data were expressed as mean \pm s.D. Gestational profiles were subjected to a one-way ANOVA followed by pairwise multiple comparison procedures (Student-NewmanKeuls method) to determine differences between groups. Data from the tubal ligation study were analyzed by two-way ANOVA followed by pairwise multiple comparison procedures. Statistical analysis was carried out using SigmaStat version 2.01 (Jandel Corp., San Rafael, CA, USA) with the level of significance for comparison set at $P<0.05$.

\section{Declaration of interest}

The authors declare that there is no conflict of interest that could be perceived as prejudicing the impartiality of the research reported.

\section{Funding}

This study was supported by a grant from the CIHR\# 37775 . 


\section{Acknowledgements}

The authors thank Ms Anna Dorogin for expert technical assistance in tissue collection, processing, and sectioning. We also thank Dr T Ansari and Ms Kate Widdows for their stimulating discussions and help with the stereology software.

\section{References}

Bailey KM \& Liu J 2008 Caveolin-1 up-regulation during epithelial to mesenchymal transition is mediated by focal adhesion kinase. Journal of Biological Chemistry 283 13714-13724.

Campbell L, Gumbleton M \& Ritchie K 2001 Caveolae and the caveolins in human disease. Advanced Drug Delivery Reviews 49 325-335.

Couet J, Belanger MM, Roussel E \& Drolet MC 2001 Cell biology of caveolae and caveolin. Advanced Drug Delivery Reviews 49 223-235.

Csapo A, Erdos T, De Mattos CR, Gramss E \& Moscowitz C 1965 Stretchinduced uterine growth, protein synthesis and function. Nature 207 1378-1379.

Douglas AJ, Clarke EW \& Goldspink DF 1988 Influence of mechanical stretch on growth and protein turnover of rat uterus. American Journal of Physiology 254 E543-E548.

Gabella G 1990 Hypertrophy of visceral smooth muscle. Anatomy and Embryology 182 409-424.

Goldspink DF \& Douglas AJ 1988 Protein turnover in gravid and nongravid horns of uterus in pregnant rats. American Journal of Physiology 254 E549-E554.

Gundersen HJ \& Jensen EB 1985 Stereological estimation of the volumeweighted mean volume of arbitrary particles observed on random sections. Journal of Microscopy 138 127-142.

Gundersen HJ \& Jensen EB 1987 The efficiency of systematic sampling in stereology and its prediction. Journal of Microscopy 147 229-263.

Harkness ML \& Harkness RD 1954 The collagen content of the reproductive tract of the rat during pregnancy and lactation. Journal of Physiology 123 492-500.

Hayashi K, Matsuda S, Machida K, Yamamoto T, Fukuda Y, Nimura Y, Hayakawa T \& Hamaguchi M 2001 Invasion activating caveolin-1 mutation in human scirrhous breast cancers. Cancer Research 61 2361-2364.

van der Heijden FL \& James J 1975 Polyploidy in the human myometrium. Zeitschrift für Mikroskopisch-Anatomische Forschung 89 18-26.

Jaffer S, Shynlova O \& Lye SJ 2009 mTOR mediates estrogen-induced myometrial proliferation during pregnancy. Endocrinology 150 4672-4680.

Johansson B 1984 Different types of smooth muscle hypertrophy. Hypertension 6 III64-III68.

Koseki Y \& Fujimoto GI 1974 Progesterone effects contrasted with 17betaestradiol on DNA synthesis and epithelial nuclear proliferation in the castrate rabbit uterus. Biology of Reproduction 10 596-604.

Lindner P, Mattiasson A, Persson L \& Uvelius B 1988 Reversibility of detrusor hypertrophy and hyperplasia after removal of infravesical outflow obstruction in the rat. Journal of Urology 140 642-646.

Macphee DJ \& Lye SJ 2000 Focal adhesion signaling in the rat myometrium is abruptly terminated with the onset of labor. Endocrinology 141 274-283.
Martin L, Finn CA \& Trinder G 1973 Hypertrophy and hyperplasia in the mouse uterus after oestrogen treatment: an autoradiographic study. Journal of Endocrinology 56 133-144.

Mayhew TM 1989 Stereological studies on rat spinal neurons during postnatal development: estimates of mean perikaryal and nuclear volumes free from assumptions about shape. Journal of Anatomy $\mathbf{1 6 2}$ 97-109.

Mouton PR 2002 Principles and practices of unbiased stereology: an introduction for bioscientists. Baltimore: The John Hopkins University Press.

Nielsen KK, Andersen CB, Petersen LK, Oxlund H \& Nordling J 1995 Morphological, stereological, and biochemical analysis of the mini-pig urinary bladder after chronic outflow obstruction and after recovery from obstruction. Neurourology and Urodynamics 14 269-284.

Ou CW, Chen ZQ, Qi S \& Lye SJ 1998 Increased expression of the rat myometrial oxytocin receptor messenger ribonucleic acid during labor requires both mechanical and hormonal signals. Biology of Reproduction 59 1055-1061.

Owens GK 1989 Control of hypertrophic versus hyperplastic growth of vascular smooth muscle cells. American Journal of Physiology 257 H1755-H1765.

Park H, Go YM, Darji R, Choi JW, Lisanti MP, Maland MC \& Jo H 2000 Caveolin-1 regulates shear stress-dependent activation of extracellular signal-regulated kinase. American Journal of Physiology. Heart and Circulatory Physiology $278 \mathrm{H} 1285-\mathrm{H} 1293$.

Rothberg KG, Heuser JE, Donzell WC, Ying YS, Glenney JR \& Anderson RG 1992 Caveolin, a protein component of caveolae membrane coats. Cell 68 673-682.

Shynlova O, Mitchell JA, Tsampalieros A, Langille BL \& Lye SJ 2004 Progesterone and gravidity differentially regulate expression of extracellular matrix components in the pregnant rat myometrium. Biology of Reproduction 70 986-992.

Shynlova O, Oldenhof A, Dorogin A, Xu Q, Mu J, Nashman N \& Lye SJ 2006 Myometrial apoptosis: activation of the caspase cascade in the pregnant rat myometrium at midgestation. Biology of Reproduction $\mathbf{7 4}$ 839-849.

Sunaga N, Miyajima K, Suzuki M, Sato M, White MA, Ramirez RD, Shay JW, Gazdar AF \& Minna JD 2004 Different roles for caveolin-1 in the development of non-small cell lung cancer versus small cell lung cancer. Cancer Research 64 4277-4285.

Wei Y, Yang X, Liu Q, Wilkins JA \& Chapman HA 1999 A role for caveolin and the urokinase receptor in integrin-mediated adhesion and signaling. Journal of Cell Biology 144 1285-1294.

Wu X, Morgan KG, Jones CJ, Tribe RM \& Taggart MJ 2008 Myometrial mechanoadaptation during pregnancy: implications for smooth muscle plasticity and remodelling. Journal of Cellular and Molecular Medicine 12 1360-1373.

Received 24 June 2009

First decision 23 July 2009

Accepted 22 September 2009 